\title{
Continuous and Discrete Fourier Transforms of Steplike Waveforms
}

\author{
WILLIAM L. GANS, MEMBER, IEEE, AND NORRIS S. NAHMAN, FELLOW, IEEE
}

\begin{abstract}
A steplike waveform which has attained its final value is converted into a duration-limited one which preserves the spectrum of the original waveform and is suitable for discrete Fourier transform (DFT) computations. The method, which is based upon the response of a time-invariant linear system excited by a rectangular pulse of suitable duration, is first applied to continuous waveforms and then to discrete (sampled) waveforms. For completeness, the difference (error) between the spectra of a continuous waveform and a discrete representation of it are reviewed.
\end{abstract}

\section{INTRODUCTION}

$\mathrm{T}$ HE PURPOSE of this paper is to provide the original derivation of one simple method for converting either a continuous or discrete (sampled) steplike waveform which has attained its final value into a duration-limited one. This type of conversion is required to produce a periodic waveform which can be represented by a finite or infinite Fourier series, as the case may be.

If a discrete Fourier transform (DFT) method is to be used to analyze a continuous periodic waveform, say with the fast Fourier transform (FFT), a discrete representation of the continuous waveform must be used. Because the discrete representation of the continuous waveform is approximate, its use introduces errors. For completeness, a review of the error introduced by the FFT analysis of a periodic continuous waveform is included.

The method described here was first suggested by the second author, in 1971, to one of his National Bureau of Standards co-workers, Mark Synder-Stonebraker. At that time it was used to obtain the complex Fourier spectrum of steplike waveforms obtained from simulation studies of certain timedomain metrology experiments. The method has been extensively developed and used in computer-controlled experiments by the first author [1], [2] and was reported to a limited European audience in 1978. ${ }^{1}$ Subsequently, it has been referred to as the Gans method [3], [4].

Furthermore, the method presented here is not the only one available for converting the steplike waveform into a duration-limited waveform. There are others such as differentiation

Manuscript received March 2, 1981; revised December 7, 1981 and March $1,1982$.

The authors are with the National Bureau of Standards Electromagnetic Technology Division, National Bureau of Standards, Boulder, CO 80303.

1 Centre National d'Etudes des Telecommunications (CNET) 1978 Summer School, Theory and Practice of Time-domain Measurements, $\mathbf{N}$. S. Nahman, Principal Professor; Lannion, France, July 1978. (Ecole d'Ete du Tregor, Session 1978 Théorie et Pratique des Mesures Temporelles, Lannion, France.) Gans, W. L., "Lectures G1, G2, and G3," CNET 1978 Summer School.

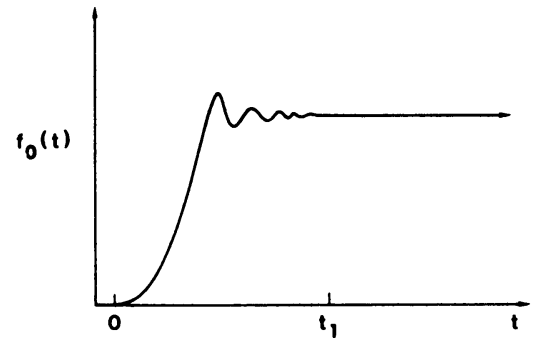

(a)

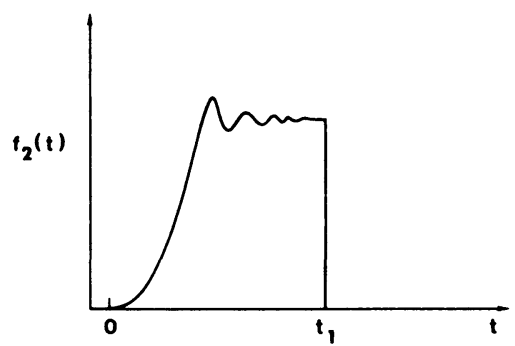

(b)

Fig. 1. The steplike waveform $f_{0}(t)$ attains its final value at $t_{1} \cdot f_{2}(t)$ is $f_{0}(t)$ truncated at $t=t_{1}$.

of the steplike waveform [5], and the addition of a ramp function [6]. Both of these methods may have an advantage (to the user) in that for DFT implementation (FFT or otherwise) they only require a time window of $t_{1}$ seconds ( $N$ points spread over $t_{1}$ seconds). On the other hand, our method requires a time window of $2 t_{1}$ seconds ( $2 N$ points over $2 t_{1}$ seconds) to provide the same number of spectral values ( $N$ values) for the spectrum of the original discrete steplike waveform $\hat{f}_{0}(t)$. Whichever method is used, however an awareness of all the potential pitfalls and sources of error is required to ensure accurate and meaningful Fourier transformation results.

The discussion is divided into three parts: 1) the conversion of a continuous steplike waveform, 2) the conversion of a discrete steplike waveform, and 3) a review of the error introduced by approximating a continuous waveform with a discrete (sampled) one.

\section{Conversion of a Continuous Steplike WAVEFORM}

Consider a continuous causal steplike waveform, $f_{0}(t)$, which is zero for time less than zero and reaches its final value, $K(K \neq 0)$ at some time, $t_{1}$, and remains there for increasing time, Fig. 1(a). 


$$
f_{0}(t)= \begin{cases}0, & t<0 \\ f_{1}(t), & 0<t<t_{1} \\ K, & t>t_{1} .\end{cases}
$$

Because $f_{0}(t)$ is zero for $t<0$, it is a causal function and as such its spectrum satisfies the Paley-Wiener criterion [7, p. 130] and is not band limited. For a function to be band limited, its spectrum must occupy a finite region of the spectral domain which violates the Paley-Wiener criterion.

It is desired to convert $f_{0}(t)$ into a duration-limited waveform which corresponds to one period of a periodic waveform, thus allowing the duration-limited waveform to be represented by a Fourier series. Also, it is desirable to use a conversion method which provides an easy technique for recovering the spectrum of $f_{0}(t)$ from that of a converted waveform. For example, an unsatisfactory way of converting the steplike waveform would be to abruptly truncate it at $t=t_{1}$, to obtain a pulselike waveform, $f_{2}(t)$, Fig. 1(b) [6].

Physically, this method is unsatisfactory because the abrupt truncation introduces the frequency components of the ideal step function, $K u(t)$, where

$$
u(t)= \begin{cases}0, & t<0 \\ 1, & t>0\end{cases}
$$

Mathematically, the Fourier transform [7] of the truncated waveform is given by

$$
\begin{aligned}
F_{2}(j \omega) & =\int_{-\infty}^{\infty} f_{2}(t) e^{-j \omega t} d t \\
& =\int_{0}^{\infty}\left[f_{0}(t)-K u\left(t-t_{1}\right) e^{-j \omega t} d t\right. \\
& =F_{0}(j \omega)-K\left[\pi \delta(\omega)+\frac{1}{j \omega}\right] \\
& =F_{0}(j \omega)-F_{e}(j \omega)
\end{aligned}
$$

where the first term of the result (3) is the desired transform, and the second term is the erroneous component introduced by the step function. The transform (3) can only be used to determine $F_{0}(j \omega)$ when a corrective action is taken to remove the error term $F_{e}(j \omega)$ from the transform $F_{2}(j \omega)$.

To avoid having to correct the resultant Fourier transform after the fact, all that is required is to turn off $f_{0}(t)$ in a practical physical manner. For example, $f_{0}(t)$ may be turned off in the same way as the response of a certain class of time-invariant linear systems (low-pass filters) to an ideal rectangular pulse, $f_{3}(t)$, if the filter response has attained the final value $K$. This rectangular-pulse response is shown in Fig. 2 and is expressed as

$$
f_{3}(t)=f_{0}(t)-f_{0}\left(t-t_{1}\right) u\left(t-t_{1}\right)
$$

The assumption that the filter response has attained its final value $K$ and remains there before the ideal rectangular driving pulse switches to zero at some finite time cannot be satisfied exactly by a dynamic time-invariant linear system because such a system contains energy storage elements which reach their final values only at $t=\infty$. However, this assumption is
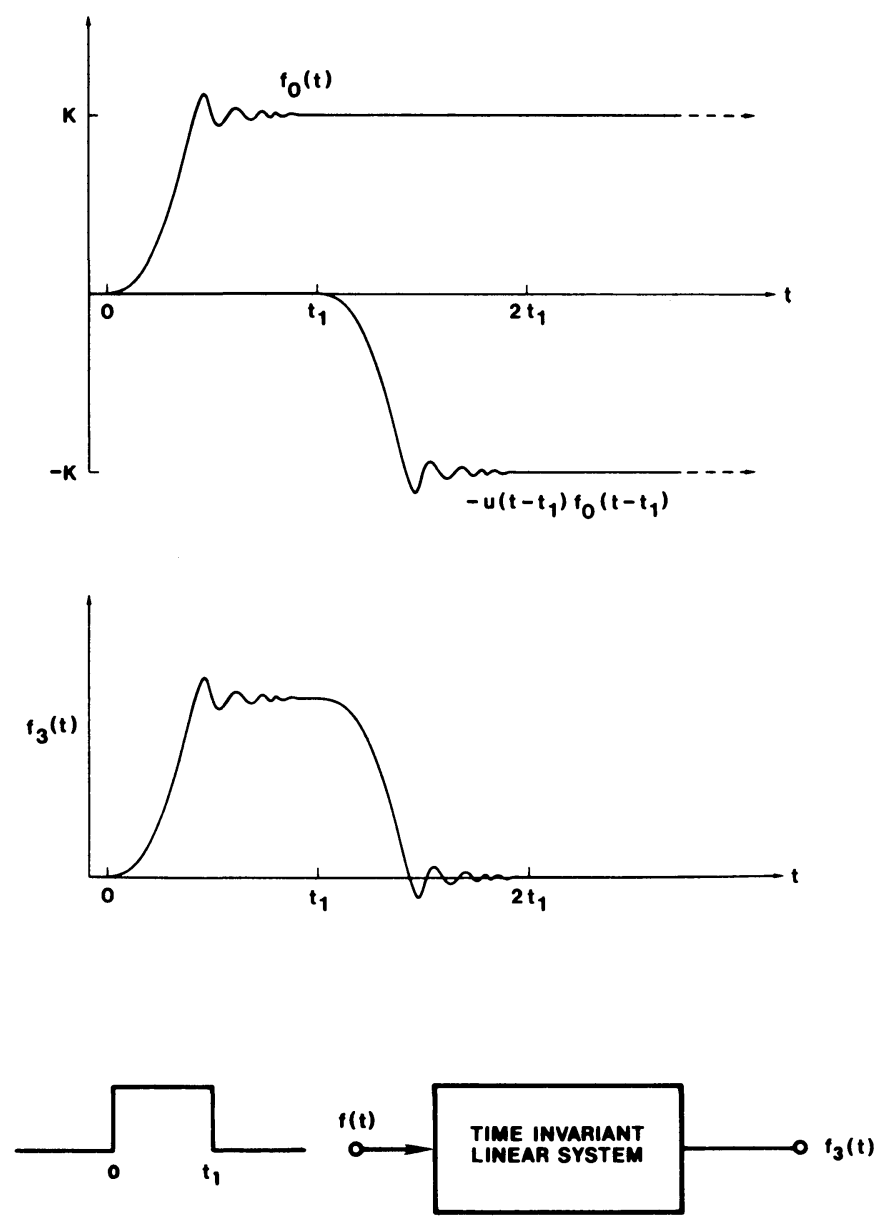

Fig. 2. The response to a rectangular pulse by a time-invariant linear system whose step response is $f_{0}(t) . f_{3}(t)$ is the sum of $f_{0}(t)$ and $-u\left(t-t_{1}\right) f_{0}$ $\left(t-t_{1}\right)$.

acceptable in a practical sense because after some time, say $t_{1}$ as said above, the change in the response waveform is negligible and cannot be discerned. This is a fundamental assertion in every experimental investigation which seeks to characterize a time-invariant linear system by time-domain measurements. Therefore, since the method is to be applied to such physical waveforms, the assumption is acceptable. A similar assumption of a finite data domain must also be made in system characterization using frequency-domain (steady-state) data which would theoretically require data from $\omega=-\infty$ to $\omega=\infty$.

The Fourier transform of $f_{3}(t)$ is given by

$$
\begin{aligned}
F_{3}(j \omega) & =\int_{-\infty}^{\infty} f_{3}(t) e^{-j \omega t} d t=\int_{0}^{2 t_{1}} f_{3}(t) e^{-j \omega t} d t \\
& =F_{0}(j \omega)\left[1-e^{-j \omega t_{1}}\right] \\
& =F_{0}(j \omega)\left[1-\left(\cos \omega t_{1}-j \sin \omega t_{1}\right)\right] \\
& =F_{0}(j \omega)\left[F_{4}(j \omega)\right] .
\end{aligned}
$$

$F_{0}(j \omega)$ is the desired Fourier transform, and it is multiplied by the bracketed function, $F_{4}(j \omega)$. Now consider $F_{4}(j \omega)$. When $\cos \omega t_{1}$ is -1 , the angular frequency is given by $\omega_{n}=$ $n \pi / t_{1}$ where $n$ represents all positive and negative odd integers, 

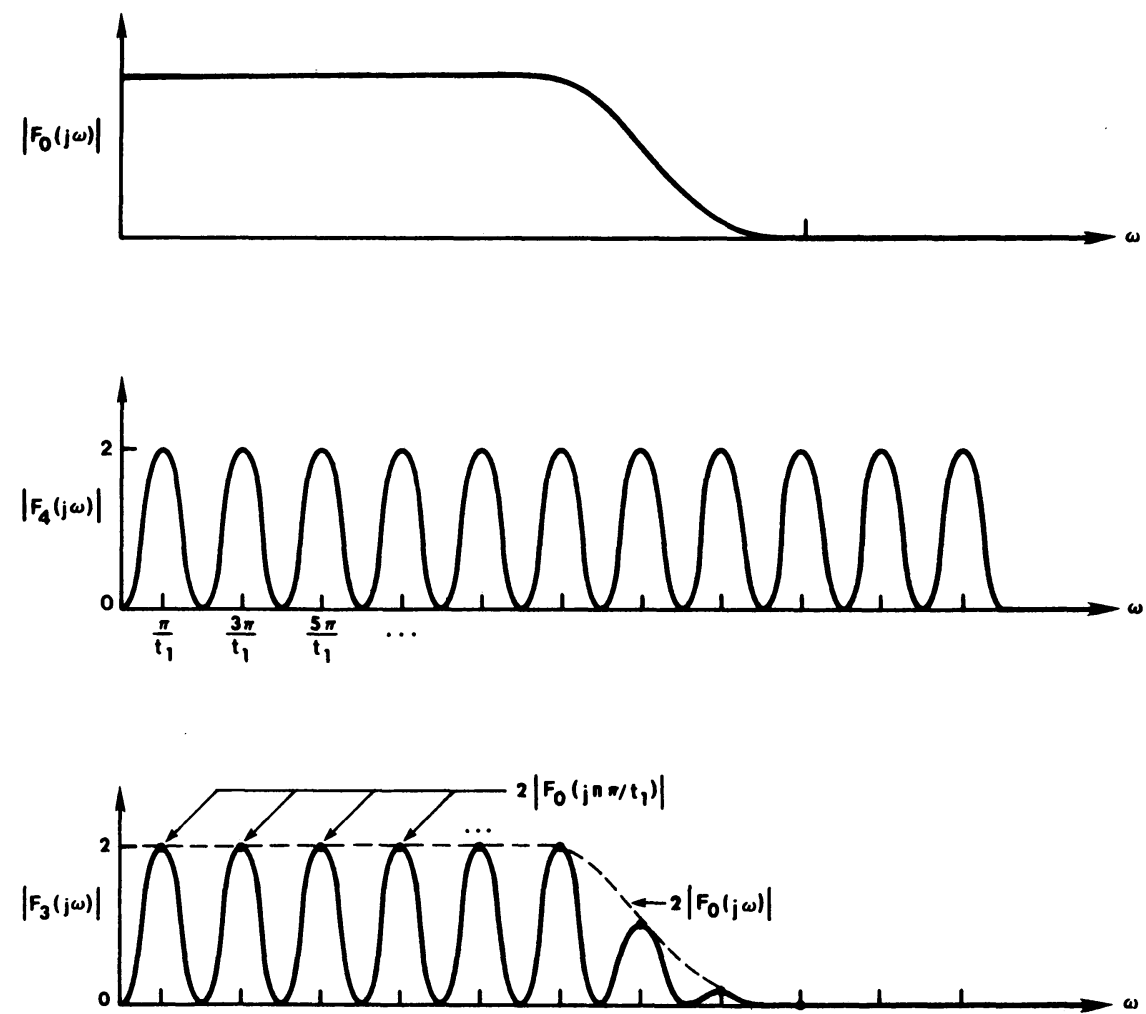

Fig. 3. The Fourier transforms of the continuous waveforms $f_{0}(t)$ and $f_{3}(t)$. The magnitudes of the Fourier transforms $\left|F_{0}(j \omega)\right|,\left|F_{4}(j \omega)\right|$, and their product $\left|F_{3}(j \omega)\right|$, which is exactly equal to twice $\left|F_{0}(j \omega)\right|$ for the discrete frequencies $\omega_{n}=n \pi t_{1} / 2, n=1,3,5, \cdots$. The spectra are only shown for $\omega \geq 0$ where $|F(j \omega)|=|F(-j \omega)|$.

and $\sin \omega_{n} t_{1}$ is zero. Consequently

$$
F_{3}\left(j \omega_{n}\right)=2 F_{0}\left(j \omega_{n}\right), \quad n= \pm 1, \pm 3, \pm 5, \cdots .
$$

On the other hand, when $\cos \omega t_{1}$ is 1 , the angular frequency is $\omega_{m}=m \pi / t_{1}$, where $m$ represents zero and all positive and negative even integers. Again, $\sin \omega_{m} t_{1}$ is zero. Hence one obtains

$$
F_{3}\left(j \omega_{m}\right)=0, \quad m= \pm 0, \pm 2, \pm 4, \cdots .
$$

It is seen that for the discrete frequencies, $\omega_{n}$, the transform $F_{3}(j \omega)$ is exactly twice the desired transform $F_{0}(j \omega),(6)$. This result can be represented graphically by considering the magnitude of $F_{3}(j \omega)$

$$
\left|F_{3}(j \omega)\right|=\left|F_{0}(j \omega)\right|\left|F_{4}(j \omega)\right|=2\left|F_{0}(j \omega)\right|\left|\sin \left(\omega t_{1} / 2\right)\right| .
$$

Fig. 3 shows sketches of $\left|F_{0}(j \omega)\right|,\left|F_{4}(j \omega)\right|$, and their product, $\left|F_{3}(j \omega)\right|$. Also, for the discrete frequencies, $\omega_{m}$ and $\omega_{n}$, the phase angle of $F_{3}(j \omega)$ is zero; consequently, the phase $F_{3}\left(j \omega_{m}, \omega_{n}\right)$ is that of $F_{0}\left(j \omega_{m}, \omega_{n}\right)$. The spectra in Fig. 3 are continuous, corresponding to the causal continuous functions $f_{0}(t)$ and $f_{3}(t)$. The analysis has demonstrated a method for converting the steplike waveform, $f_{0}(t)$, that has attained its final value into a duration-limited waveform, $f_{3}(t)$; the method preserves the complex spectrum of the steplike waveform only at specific known discrete frequencies which, in turn, allows the recovery of the steplike waveform's complex spectrum at those frequencies.

\section{Conversion of A Discrete Steplike WAVEFORM}

Given a discrete, steplike waveform which has attained its final value, it can be converted to a discrete duration-limited waveform. In this section the effects of approximating a continuous waveform by a discrete one do not need to be considered because the given waveform is already a discrete one. Such approximation effects are reviewed in section IV.

Proceeding in the same manner as in the continuous case, the properly truncated or converted discrete waveform of the same form as the continuous waveform (4), is the sequence

$$
\left\{f_{3}(n T)\right\}=\left\{f_{0}(n T)\right\}-\left\{f_{0}(n t-n T)\right\}
$$

where $\left\{f_{0}(n T)\right\}$ is the original steplike sequence. The DFT of the sequence $\left\{f_{3}(n T)\right\}$ is

$$
\begin{array}{r}
\left\{F_{D 3}(\Omega k)\right\}=\left\{F_{D 0}(\Omega k)\right\}-\left\{e^{-j \Omega k N T} F_{D 0}(\Omega k)\right\} \\
=\sum_{n=0}^{2 N-1} f_{0}(n T) e^{-j \Omega T n k}\left(1-e^{-j \Omega T k N}\right), \\
k=0,1, \cdots, 2 N-1 .
\end{array}
$$

Notice that this sequence has $2 N-1$ values over a time interval of $2 t_{1}$, Fig. 4 . The term $1-\exp (-j \Omega T k N)$ corresponds to $F_{4}(j \omega)$ in the continuous case. The magnitude $\mid F_{D 4}$ $(\Omega k) \mid$ :

$$
\left|F_{D 4}(\Omega k)\right|=\left|1-e^{-j \Omega T k N}\right|=2|\sin \pi k / 2| .
$$



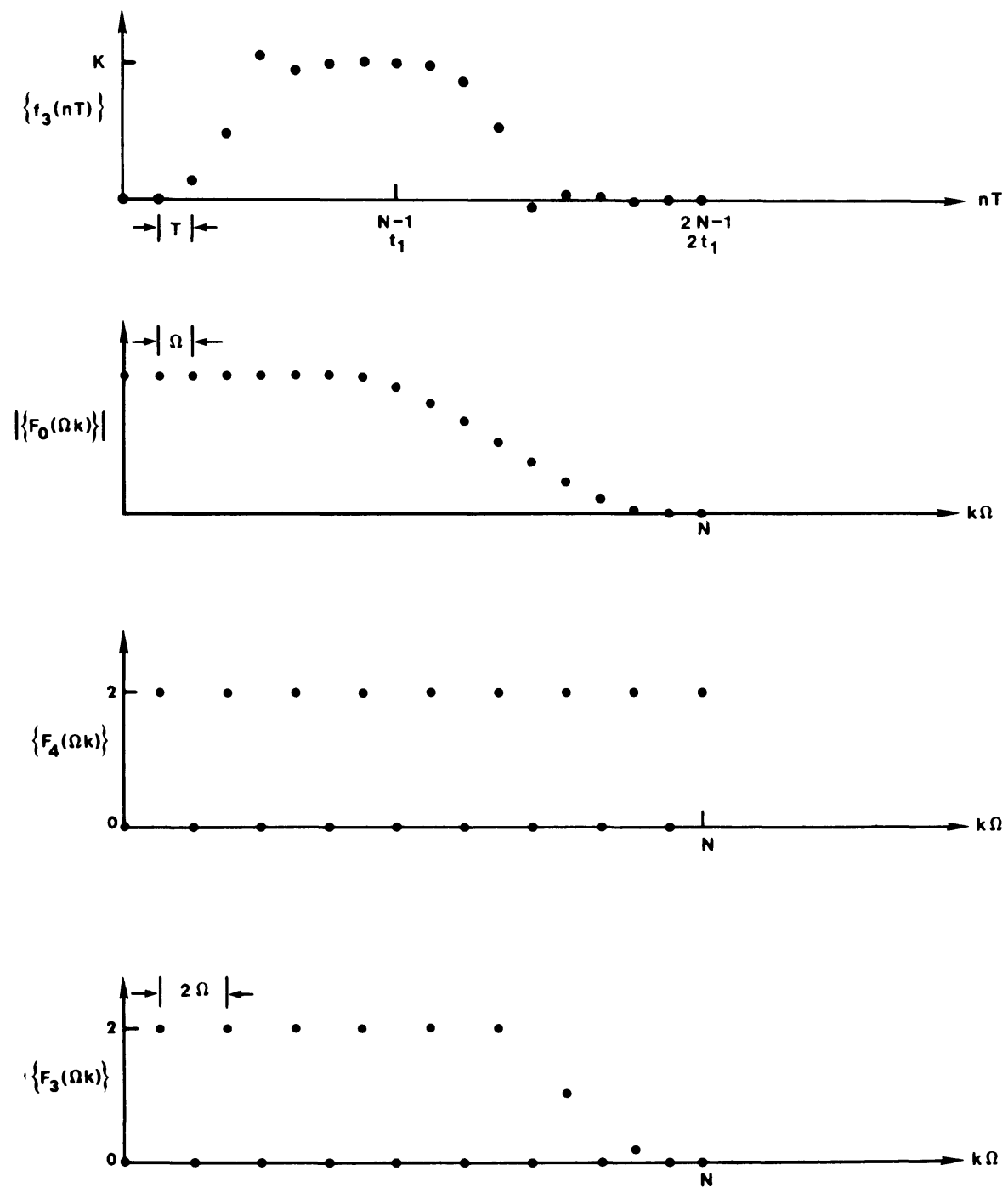

Fig. 4. The transformed discrete waveform sequence $\left\{f_{3}(n T)\right\}$ and its discrete spectral sequences. The spectral sequence $\left.\left\{F_{3} \Omega k\right)\right\}$ provides $N$ values of the spectrum corresponding to the original steplike sequence $\left\{f_{0}(n T)\right\}$. The frequency spacing, $2 \Omega$, corresponds to $N$ samples or values in $\left\{f_{0}(n T)\right\}$ spaced over $t_{1}$ seconds. The spectrum magnitudes are shown only out to the folding abscissa value (folding frequency).

weights the spectrum of $F_{D 0}(\Omega k)$ in the same manner as in the continuous case, Fig. 4; similarly, the phase angle of $F_{D 4}(\Omega k)$ is zero. In Fig. 4 notice that the resultant discrete spectrum sequence $F_{3}(\Omega k)$ provides $N$ nonzero values of the discrete step-like waveform's spectral sequence $F_{0}\left(m \Omega_{1}\right)$ where $\Omega_{1}=$ $2 \Omega$ and $m=0,1,2, \cdots, N-1$. This corresponds to $N$ samples over a time interval (window) of $t_{1}$ seconds as was specified for the original steplike waveform.

It is important to understand that the results obtained above, (8) and (10), are exact results for the continuous and discrete waveforms, $f_{0}(t)$ and $\left\{f_{0}(t)\right\}$, respectively. However, because 1) $f_{0}(t)$ and $\left\{f_{0}(t)\right\}$ are different functions of time, and 2$)$ the continuous Fourier transformation (CFT) and the DFT are two different transformations, the transforms, the CFT $F_{0}(j \omega)$ and the DFT $\left\{F_{D 0}(n T)\right\}$ are not equal; consequently, the CFT's $F_{3}(j \omega)$ and $F_{4}(j \omega)$ and the DFT's $\left\{F_{D 3}(n T)\right\}$ and $\left\{F_{D 4}(n T)\right\}$ also are not equal, respectively. Moreover, the inequality is fundamental because one transform is a continuous function while the other one is a discrete function.

To emphasize the difference between the continuous and discrete transformation results, a review of the discrete waveform approximation to a continuous waveform is given in the next section.

\section{REVIEW OF THE DISCRETE WAVEFORM APPROXIMATION TO A CONTINUOUS WAVEFORM}

The discussion which ensues in this section is relevant to the errors introduced by the discrete (sampled) representation of continuous waveforms. Because physical (analog) signals are continuous, discrete representations (approximations) of them are used to implement DFT analyses; and accordingly, the resultant spectrum differs from that of the continuous waveform. The discussion is included here because it is likely that 
these errors could be assumed to be negligible without a prior analysis in the application of DFT analysis to a physical waveform.

If a steplike continuous causal waveform, $f_{0}(t)$, is approximated by a sampled-data version, $\hat{f}_{0}(t)$, the approximate causal discrete waveform can be expressed as

$$
\hat{f}_{0}(t)=T \sum_{n=0}^{N-1} f_{0}(n T) \delta(t-n T), \quad 0<t<t_{1}
$$

where $N$ and $T$ are the number of samples and the sampling interval, respectively. ${ }^{2}$ Similarly, the causal duration limited waveform, $f_{3}(t)$, is approximated by

$$
\hat{f}_{3}(t)=T \sum_{n=0}^{2 N-1} f_{3}(n T) \delta(t-n T), \quad 0<t<2 t_{1} .
$$

It is important to keep in mind that the causal discrete (sampled) waveform $\hat{f}(t)$ is an approximation for certain purposes to the causal continuous waveform, $f(t)$; the two waveforms are not equivalent in the time domain or in the frequency domain. For Fourier transform analysis or any other integral operation purposes, it is convenient to represent a discrete waveform in the form of (9). However, this discrete waveform is just a finite sequence of values and as such is transformable by the DFT. Denoting the sequence of $N$ values of $f_{0}(t)$ as $f_{0}(n T)$, the DFT is

$$
\begin{aligned}
\operatorname{DFT}\left\{f_{0}(n T)\right\}=\left\{F_{D 0}(\Omega k)\right\}= & \sum_{n=0}^{N-1} f_{0}(n T) e^{-j(2 \pi / N T) T n k}, \\
& k=0,1,2, \cdots, N-1
\end{aligned}
$$

where $\Omega=2 \pi / N T$ which is the frequency spacing of the resultant transform sequence. It can be shown that the DFT (14), can be expressed in terms of the CFT of $\hat{f}_{0}(t)[7, \mathrm{p} .179]$. The CFT of (12) is given by

$$
\hat{F}_{0}(j \omega)=\int_{-\infty}^{\infty} \hat{f}_{0}(t) e^{-j \omega t} d t
$$

Expressing (14) in terms of the result from (15) gives the sequence

$$
\begin{aligned}
\left\{F_{D 0}(\Omega k)\right\}= & \left\{F_{0}(2 \pi k / N T\}+\{\epsilon\},\right. \\
& k=0,1,2, \cdots, N-1
\end{aligned}
$$

where $\{\epsilon\}$ is the error between the DFT, $F_{D 0}(\Omega k)$, and the sequence obtained from the CFT, $F_{0}(j \omega)$, evaluated at the discrete frequencies $\omega_{n}=2 \pi n / N T$, and

\footnotetext{
2 The notation and approach follow those in [7, p. 177].
}

$$
\begin{aligned}
\{\epsilon\}=\sum_{\substack{n=-\infty \\
n \neq 0}}^{\infty} F_{0}\left(\frac{2 \pi k}{N T}-\frac{2 \pi n}{T}\right), & \\
& k=0,1,2, \cdots, N-1 .
\end{aligned}
$$

An example of this error sequence is given in [7, p. 188].

The roint being made here is that for a causal waveform, i.e., a non-band-limited waveform, the DFT can never be exactly equal to the CFT, $F_{0}(j \omega)$, evaluated at discrete values of frequency, $2 \Omega k / N T$; the error $\{\epsilon\}$ (or aliasing) is always present but may be reduced to any desired amount by decreasing the sample spacing, $T$.

\section{SUMmary}

A method has been demonstrated for converting either a continuous or discrete (sampled) steplike waveform which has attained its final value into a duration-limited waveform. The method is based upon the practical physical response of a causal time-invariant linear system to a rectangular pulse of sufficient duration. The complex spectrum of the steplike waveform is preserved at known discrete frequencies in the complex spectrum for the duration-limited waveform; this, in turn, allows the recovery of the steplike waveform's complex spectrum.

Furthermore, the distinction between the continuous Fourier transformation and the discrete Fourier transformation has been reviewed. This has been done to emphasize the point that a causal continuous (physical) waveform in only approximated by a discrete (sampled) waveform and consequently their respective complex spectra always differ to some degree, i.e., aliasing is always present to some degree.

\section{REFERENCES}

[1] W. L. Gans, "A time domain network analyzer for microwave measurements," M.S.E.E. thesis, Univ. of Colorado, Boulder, Spring 1975.

[2] W. L. Gans, and J. R. Andrews, "Time domain automatic network analyzer for measurement of RF and microwave components," National Bureau of Standards, Boulder, CO, Tech. Note 672, Sept. 1975.

[3] Y. Dutuit, "La transformation de Fourier discrete en spectroscopie temporelle," Revue de Physique Appliquee, Tome 14, pp. 939-945, Nov. 1979.

[4] J. Waldmeyer "Fast Fourier transform of steplike functions: The synthesis of three apparently different methods," IEEE Trans. Instrum. Meas., vol. IM-29, p. 36, Mar. 1980.

[5] H. A. Samulon, "Spectrum analysis of transient response curves," Proc. IRE, vol. 39, pp. 175-186, 1951.

[6] A. M. Nicolson, "Forming the fast Fourier transform of a step response in time-domain metrology," Electron. Lett., vol. 9, no. 14, pp. 317-318, July 12, 1973.

[7] C. D. McGillem, and G. R. Cooper, "Continuous and discrete signal and system analysis," New York: Holt, Rinehart, and Winston, 1974, pp. 130, 179. 